\title{
Integrated imaging - the complementary roles of radiology and nuclear medicine
}

\author{
J Warwick, ${ }^{1}$ BSC, MB ChB, FCNP (SA), MMed, PhD; J Lotz, ${ }^{2}$ MA, MSc, MMed, FCRad (SA), FRCR \\ ${ }^{1}$ Division of Nuclear Medicine, Tygerberg Academic Hospital and Stellenbosch University, Cape Town, South Africa \\ ${ }^{2}$ Division of Radiodiagnosis, Tygerberg Academic Hospital and Stellenbosch University, Cape Town, South Africa
}

Corresponding author: J Warwick (jw@sun.ac.za)

This article was first published in the August 2013 issue of Continuing Medical Education (Vol. 31, Issue 8), and is reproduced here because of its topicality and desirability of reaching a broader audience.

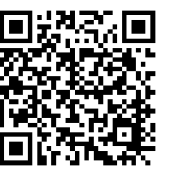

Diagnostic imaging is a dynamic field, which has evolved rapidly during the past few decades. New modalities have developed and research techniques have migrated into clinical practice. Imaging has seen a greater integration of various modalities, defining the entity of diagnostic clinical imaging sciences.

Nuclear medicine has undergone a metamorphosis over the past 30 years. Established scintigraphic techniques for detecting breaches in the blood-brain barrier and liver masses has been replaced by ultrasound, computerised tomography (CT) and magnetic resonance imaging (MRI). Simultaneously, there has been significant growth in the use of positron emission tomography (PET), sentinel node imaging, targeted radiotherapy and several molecular imaging techniques.

Radiology has experienced exponential growth with regard to helical multi-slice CT scanning using multiple-row detector arrays for the simultaneous collection of data at different slice locations, allowing rapid acquisition of large datasets of longitudinal volumes. The optimal MRI field strength in general clinical practice is 1.5 tesla (T), while $3 \mathrm{~T}$ allows mapping of cerebral cortical function by utilising blood oxygen level-dependent (BOLD) imaging. In vivo spectroscopy monitors neuronal viability, cell membrane proliferation and energy metabolism. Current clinical $3 \mathrm{~T}$ products have been developed to fit within the physical constraints of $1.5 \mathrm{~T}$ platforms by using compact magnets with small fringe fields. Magnets with field strengths of $7 \mathrm{~T}$ and even higher are in experimental use. ${ }^{[1]}$

An important influence on medical imaging since the beginning of the 21st century has been the increasing availability of hybrid imaging systems. These systems enable a more integrated approach, and define the unitary entity of diagnostic clinical imaging sciences. The concept of hybrid imaging is simple - PET or a single photon emission computed tomography (SPECT) scanner is integrated with a CT scanner on a single platform. The patient is examined using both techniques in immediate succession without any positional changes. Images are fused and displayed by dedicated software. Detailed anatomical imaging combined with the high sensitivity of functional information provides much more information than would be provided by the two individual techniques. The composite images are also generally better appreciated by clinicians.

CT scanners on integrated platforms are mostly high-quality multislice diagnostic units. Adding contrast enhancement affords the potential for performing a single comprehensive diagnostic study. PET/ MRI, a recent development, is currently being clinically evaluated.
This article highlights clinical scenarios where radiology and nuclear medicine are combined to address specific clinical problems. An exhaustive list of these problems is inapplicable, as unique diagnostic dilemmas continually occur. In our experience, these are best addressed by continuous interaction and regular interdisciplinary meetings between the two disciplines.

\section{Oncology}

Due to its high sensitivity, PET- using the glucose analogue [F-18] flourodeoxyglucose (FDG) - is transforming the diagnosis and management of many malignancies. Integrated PET/CT studies enhance both accuracy and logistic efficiency. Abnormal FDG uptake helps the radiologist to scrutinise areas of subtle anatomical changes, often increasing specificity (Fig. 1). CT also enhances specificity by localising FDG uptake to physiological sites, such as brown fat, which on PET alone can mimic nodal disease. Similarly, the limitations inherent in the ability of FDG-PET to distinguish a neoplasm from inflammation are sometimes improved by CT patterns that are more suggestive of either inflammation or a neoplasm. PET generally has excellent sensitivity, while CT detects small lung nodules more accurately.

Size-based lymph node evaluation for metastatic involvement (short axis $>10 \mathrm{~mm}$ ) and morphology has a low sensitivity and specificity. FDG-PET frequently demonstrates small nodes that are not benign and large nodes that are not metastatic (Fig. 1). FDG uptake has the ability to distinguish residual tumour activity from scar tissue in residual masses after chemotherapy. ${ }^{[2]}$

\section{Neuro-endocrine tumours}

An integrated approach is essential in the assessment of neuroendocrine tumours (NETs). The final diagnostic analysis combines functional, morphological and positional information. A detailed radiological work-up is only possible once a functional lesion has been localised. Localisation of well-differentiated NETs is performed by using labelled analogues of somatostatin, or molecules concentrated by NETs. ${ }^{[3]}$ Staging and planning of targeted radiotherapy require functional techniques, while anatomical imaging is important for monitoring disease and planning of surgery (Fig. 2).

\section{Musculoskeletal imaging}

Bone scintigraphy is a first-line technique for surveying the entire skeleton for metastases in patients with cancer of the breast and 
prostate, as well as small-cell lung carcinoma. While highly sensitive, the specificity is reduced by numerous benign processes (e.g. degenerative changes, old trauma). Here, radiology has a long-established complementary role, as local radiographs of these sites identify most benign causes of increased activity, whereas normal radiographs are suggestive of metastatic disease.

The sensitivity of MRI depends on focal loss of the T1 signal due to metastatic replacement/destruction of marrow fat cells in trabecular bone. T1 changes appear up to three months before other modalities are positive; ${ }^{[4]}$ however, MRI studies are limited by cost and a small field of view, disqualifying it as a viable screening alternative to bone scintigraphy. T1-weighted MRI sequences should be reserved for specific clinical problems, e.g. breast carcinoma with persistent lower back pain, and equivocal focal activity on a bone scan not resolved with plain radiographs. Diffusion-weighted whole-body MRI is an exciting new development with promising results for cancer detection, widely reported in the recent literature. ${ }^{[5]}$

Bone scintigraphy plays a complementary role in benign bone disease, adding sensitivity and enabling an entire skeletal survey. Most fractures are diagnosed using conventional radiographs, but bone scanning is a cost-effective and sensitive means of detecting fractures, which have a normal appearance on plain films (e.g. undisplaced, difficult sites) and insufficiency/stress fractures. Scintigraphy also allows localisation of sites causing back, ankle or foot pain. When there are subtle or multiple anatomical abnormalities, active osteoblastic activity can direct therapeutic interventions to sites requiring relief of pain (Fig. 3).

Acute osteitis in children can present challenging diagnostic dilemmas. These children often present as emergency cases, acutely ill and with raised inflammatory markers. The presenting complaint may, however, be limited to diffuse bone pain with normal erythrocyte sedimentation rate (ESR) and white blood cell (WBC) counts. Plain film examination of the affected limb should precede special investigations. Ultrasound is an excellent first-line investigation and is often diagnostic (Fig. 4). CT is an acceptable alternative, but an MRI study, when available, is definitive, showing the extent of trabecular oedema and soft-tissue involvement. Skeletal scintigraphy remains particularly valuable when MRI availability is limited, symptoms are poorly localised, or multifocal disease is suspected. ${ }^{[6]}$

\section{Soft-tissue infection}

Ultrasound is normally the first line of investigation, but MRI, with its superior soft-tissue discrimination, is the investigation of choice. Inflammatory processes are identified by a hyperintense signal on T2 STIR or post-contrast T1 fat saturation sequences. However, an altered anatomy because of previous trauma or surgery may render findings equivocal. In these situations the introduction of infection markers such as labelled WBCs, FDG, and [Ga-67] citrate assist in the evaluation of disease (Fig. 5). These tracers are specific and sensitive, demonstrating inflammatory processes before anatomical changes occur. The choice of technique depends on the suspected site and chronicity of the infection. The role of FDG-PET/CT is still being defined, but it is likely to become important in many of these clinical scenarios. ${ }^{[7]}$

\section{Pulmonary embolism}

The imaging of acute pulmonary embolism has advanced with the widespread implementation of CT pulmonary angiography (CTPA) and the emerging role of ventilation perfusion (VQ) SPECT (VQS). CTPA is based on the direct detection of emboli presenting as filling defects in affected vessels, while VQS images the functional consequences of the emboli, i.e. underperfused lung segments (Fig. 6). VQS, using new interpretation criteria, achieves excellent sensitivity and specificity with few equivocal studies, and today should be utilised by all nuclear medicine units. ${ }^{[8]}$ Comparative literature is limited and complicated by the lack of a gold standard, and confused by comparisons of CTPA with older planar VQ studies.

Both CTPA and VQS are excellent first-line investigations and their availability often determines the first choice. Specific considerations such as compromised cardiorespiratory function or structural lung disease favour the use of CTPA when both modalities are available. Similarly, a mosaic perfusion pattern in the appropriate clinical setting on highresolution CT scans is diagnostic for chronic pulmonary embolism as a cause of unexplained pulmonary hypertension. Conversely, VQS has a significantly lower radiation dose, making its use preferable in pregnancy, in patients with impaired renal function, and in patients in whom prior reactions to contrast media are suspected. Perhaps, most importantly, performing the other examination is useful in those cases where one modality is initially equivocal or discordant with the clinical setting.

\section{Acute cholecystitis}

Ultrasound is the initial imaging technique of choice in patients with suspected acute cholecystitis. This is justified in light of its widespread availability and efficacy in the majority of patients. In cases where ultrasound is difficult to interpret (e.g. technically difficult examinations, acalculous disease), hepatobiliary scintigraphy has a high sensitivity and specificity, ${ }^{[9]}$ making it an appropriate second-line investigation when clinical suspicion is high.

\section{Liver lesions}

Functional imaging with characteristic scintigraphic patterns can assist with the identification of liver lesions detected on anatomical imaging (Fig. 7). In liver lesions equivocal for metastatic disease, increased uptake of FDG on PET/CT distinguishes metastatic disease from benign lesions. Increased Tc-99m colloid uptake in a liver lesion is specific for focal nodular hyperplasia. Haemangiomas show enhancement of Tc-99m-labelled red blood cells. Developments in MRI liver contrast media have resulted in substances that mirror the biodistribution patterns of radionuclide tracers. Gadolinium chelates are distributed to the interstitial space, ferumoxides are taken up by Kupffer cells, and the new mangafodiper agents create T1 shortening after hepatocyte uptake via the $\mathrm{B}_{6}$ receptor. Exquisite anatomical detail is an added advantage, but this has significant financial implications.

\section{Residual splenic tissue}

Patients with resistant idiopathic thrombocytopenic purpura, despite splenectomy, often have continued destruction of platelets by small amounts of residual splenic tissue. This tissue is frequently difficult to detect using anatomical imaging alone. SPECT/CT using [Tc-99m]labelled damaged red blood cells is sensitive and specific for splenic tissue (Fig. 8), allowing lesions to be located and guiding their surgical removal.

\section{Renal imaging}

Ultrasound plays an essential role in many types of renal disease. The combination of ultrasound and other anatomical modalities with 
functional imaging is particularly rewarding in the renal system. Diuresis renography using Tc-99m MAG-3 clarifies the significance of hydronephrosis and/or hydro-ureter. Obstructive nephropathy can be ruled out in patients with a normal diuretic response, while reduced differential renal function, especially on serial studies, points to the need for intervention (Fig. 9).

The distinction between renal artery stenosis and renal vascular hypertension explains the different roles of angiography and captopril renography in the management of these patients. While anatomical imaging is essential for the detection and sometimes for the treatment of stenotic arterial lesions, captopril renography determines the functional significance of lesions, predicts the outcome of interventions, and detects renovascular hypertension related to microvascular disease.

Radiology and nuclear medicine have overlapping roles in the imaging of renal scarring and vesico-ureteric reflux (VUR) in children. A first-time urinary tract infection (UTI) requires ultrasound, which, if normal, obviates the need for further imaging. Children with recurrent UTIs or abnormalities on ultrasound normally require a variety of possible investigations. ${ }^{[10]}$ Dimercaptosuccinic acid (DMSA) renal scintigraphy is the most sensitive widely available technique to detect renal scarring. For VUR, a micturating cysto-urethrogram (MCUG) provides important anatomical information, while indirect cystography is a well-tolerated procedure which has a low radiation dose.

Incidental renal masses are being increasingly identified on advanced imaging modalities, more so with present-generation multi-slice CT scanners. A renal pseudotumour due to a prominent column of Bertin shows uptake of Tc-99m DMSA, while renal tumours show no uptake. ${ }^{[1]}$

\section{Interventional radiology}

The interventional radiologist may be assisted by functional imaging. The choice of an optimal site for taking a biopsy is often facilitated by $\mathrm{PET} / \mathrm{CT}$, revealing active disease processes in more accessible sites. Similarly, the use of metabolic information can reduce sampling errors.

Managing gastrointestinal bleeding is challenging when endoscopy is negative, or difficult to interpret owing to large amounts of intraluminal blood. Angiography and embolisation of bleeding sites can be assisted by localisation of gastrointestinal bleeding sites using [Tc-99m]-labelled red blood cells (Fig. 10). Even localisation limited to the major artery involved prior to angiography can assist with catheter placement.

The growing utilisation of targeted radiotherapy for palliation of inoperable liver lesions requires close collaboration between the interventional radiologist, who places the catheter in the hepatic artery, and the nuclear medicine physician, who administers the therapy, after scintigraphically confirming correct positioning.

\section{Conclusions}

Taking a complementary rather than an exclusive approach allows for the optimal utilisisation of the diverse spectrum of imaging technologies available. This philosophy is being practised increasingly with the advent of hybrid imaging, and the resulting interdependence of these disciplines. A good understanding of the strengths and weaknesses of the different modalities available is needed to achieve this goal. It is the duty of imaging specialists to develop approaches to clinical problems that are better integrated, provide improved care, diagnostic accuracy, and cost-effectiveness. The optimal utilisation of all imaging modalities, including their complementary strengths, differing availability, and cost considerations, is arguably the single most important challenge to all diagnostic imaging clinicians.

\section{Summary}

- A more integrated approach to medical imaging using radiology and nuclear medicine techniques can frequently better address specific clinical problems.

- PET/CT is generally more sensitive and specific for the detection of malignancies than either modality in isolation.

- MRI and bone scan modalities have complementary roles in musculoskeletal imaging.

- Labelled white blood cells, FDG, and [Ga-67] citrate are particularly useful for imaging soft-tissue infections when the anatomical location is altered by previous trauma or surgery.

- CTPA and VQ SPECT are both excellent first-line investigations for detecting pulmonary embolism.

- Hepatobiliary scintigraphy is a useful second-line investigation for detecting acute cholecystitis if ultrasound is equivocal.

- Characteristic scintigraphic patterns can assist with the characterisation of liver lesions detected on anatomical imaging.

- Renography clarifies the functional significance of hydronephrosis and/or hydro-ureter on anatomical imaging.

- Renal scintigraphy using Tc-99m DMSA can assist in the characterisation of incidental renal masses identified on anatomical imaging.

- PET/CT and labelled red cell studies can assist the radiologist in better directing interventional procedures.

1. Blamire AM. The technology of MRI - the next 10 years? Br J Radiol 2008;81(968):601-617. [http:// dx.doi.org/10.1259/bjr/96872829]

2. Cheson BD. Role of functional imaging in the management of lymphoma. J Clin Oncol 2010;29(14):18441854. [http://dx.doi.org/10.1200/JCO.2010.32.5225]

3. Wong KK, Waterfield RT, Marzola MC, et al. Contemporary nuclear medicine imaging of neuroendocrine tumours. Clin Radiol 2012;67(11):1035-1050. [http:/dx.doi.org/10.1016/j.crad.2012.03.019]

4. Brown AL, Middleton G, MacVicar AD, et al. T1-weighted magnetic resonance imaging in breast cancer vertebral metastases: Changes on treatment and correlation with response to therapy. Clin Radiol 1998;53(7):493-501.

5. Kwee TC, Takahara T, Ochiai R, et al. Whole-body diffusion-weighted MRI. Eur J Radiol 2009;70(3):409417. [http:dx.doi.org/10.1016/j.ejrad.2009.03.054]

6. DiPoce J, Jbara ME, Brenner AI. Pediatric osteomyelitis: A scintigraphic case-based review. Radiographics 2012;32(3):865-878. [http:dx.doi.org/10.1148/rg.323115110]

7. Basu S, Chryssikos T, Moghadam-Kia S, et al. Positron emission tomography as a diagnostic tool in infection: Present role and future possibilities. Semin Nucl Med 2009;39:36-51. [http:dx.doi.org/10.1053/j. semnuclmed.2008.08.004]

8. Bajc M, Olsson B, Palmer J, et al. Ventilation/perfusion SPECT for diagnostics of pulmonary embolism in clinical practice. J Intern Med 2008;264(4):379-387. [http://dx.doi.org/10.1111/j.13652796.2008.01980.x]

9. Alobaidi M, Gupta R, Jafri SZ, et al. Current trends in imaging evaluation of acute cholecystitis. Emerg Radiol 2004;10(5):256-258.

10. Coulthard MG. NICE on childhood UTI: Nasty processes produce nasty guidelines. BMJ 2007;335:463464.

11. Vitti RA, Maurer AH. Single photon emission computed tomography and renal pseudotumor. Clin Nucl Med 1985;10(7):501-503. 


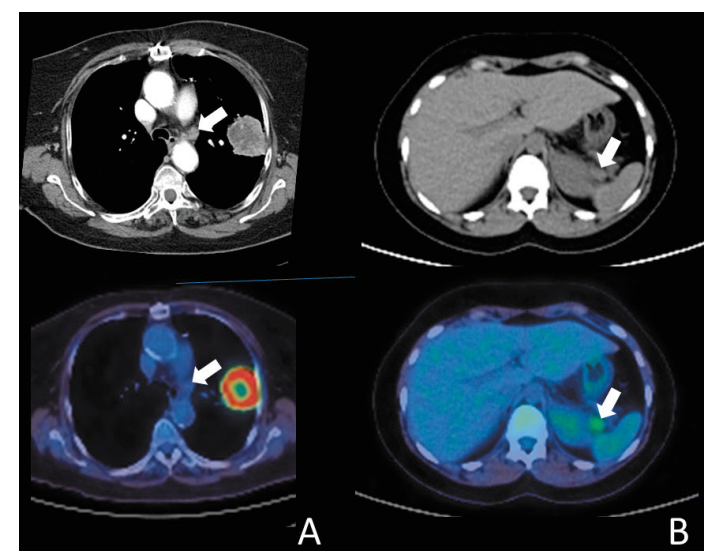

Fig. 1. Two cases of the complementary roles of PET and CT, showing (A) an enlarged lymph node identified on $C T$ in a patient with lung cancer negative on PET/CT, and (B) Hodgkin's disease in a patient with involvement of multiple lymph nodes above the diaphragm in which PET identified involvement of a single node in the splenic hilum.

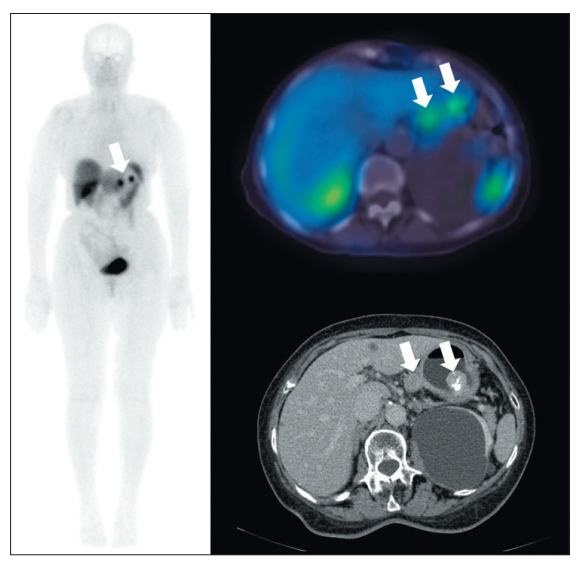

Fig. 2. Patient with a mass on the greater curve of the stomach on gastroscopy. Histology showed a NET. Whole body somatostatin-receptor imaging with [Tc-99m] Tekrotyd shows disease restricted to the stomach lesion and a lymph node. These are shown on CT with calcification in the stomach lesion, as well as incidental liver and left kidney cysts.

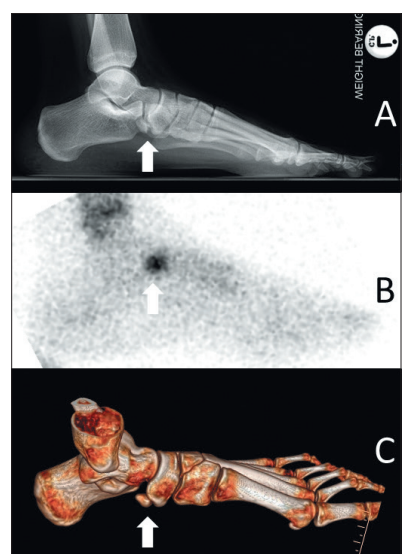

Fig. 3. (A) A patient with left foot pain, identified as having accessory navicular bones in both feet on $X$-ray. (B) A bone scan localised the pain source to the accessory navicular bone, (C) which is again demonstrated on subsequent a $3 D$ CT reconstruction.
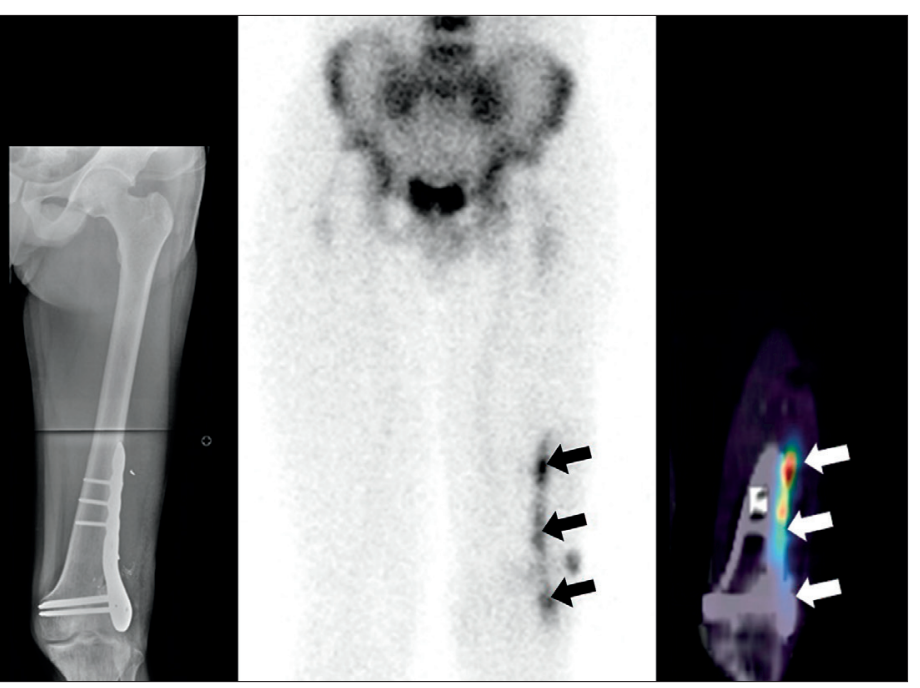

Fig. 5. A patient who required open reduction and internal fixation of a fractured left femur following a gunshot injury. The subsequent development of secondary infection is confirmed on [Tc-99m] white cell scanning. The presence of a fixation device would affect MRI and CT in this case.
Fig. 4. A longitudinal ultrasound section demonstrating a periosteum of a tibial shaft displaced by inflammatory collection in a young patient with clinical signs of infection. 


\section{TECHNICAL NOTE}

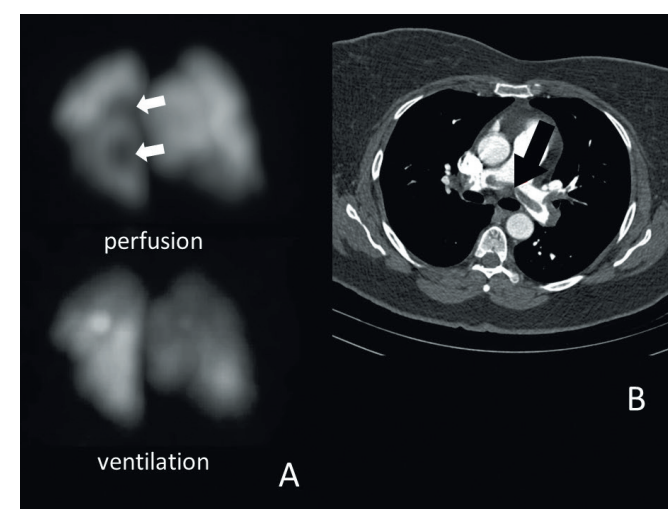

Fig. 6. Different cases showing pulmonary emboli as (A) a perfusion-ventilation mismatch using VQS, and (B) as an arterial filling defect on CTPA.

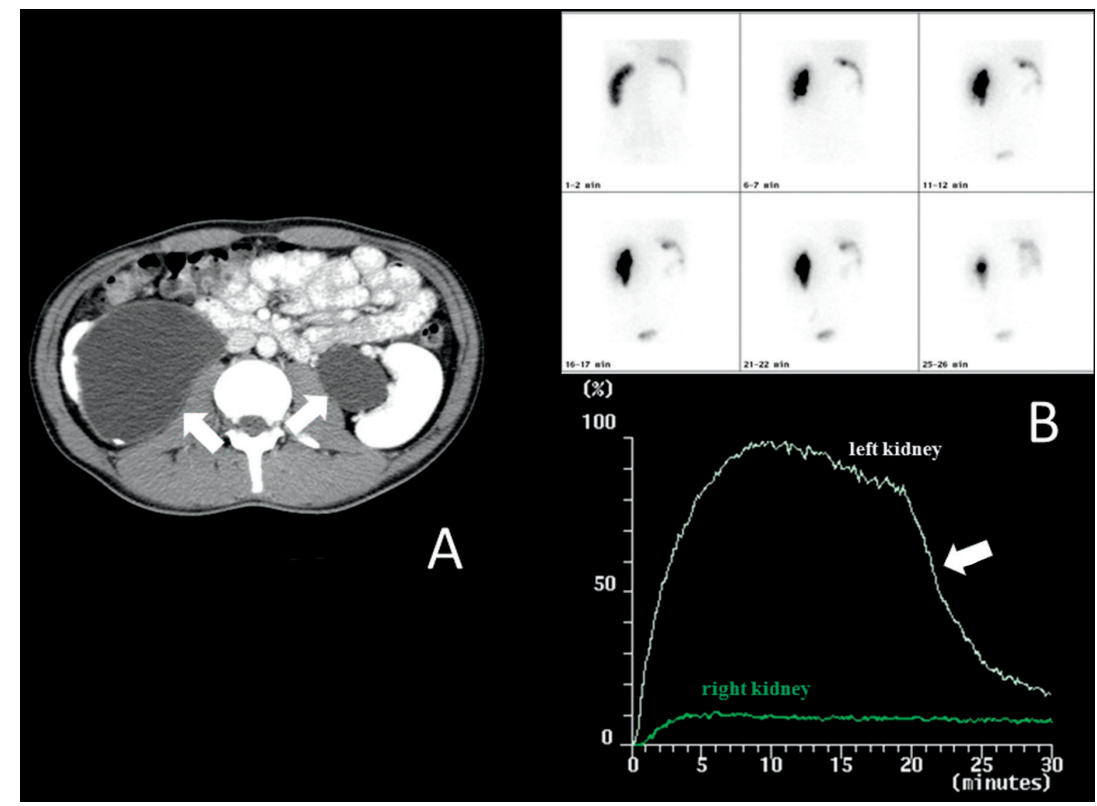

Fig. 9. Bilateral hydronephnosis on CT. The renogram shows no obstruction of the left kidney, with normal function and a good response to furosemide at $20 \mathrm{~min}$. The right kidney has a differential function of only $14 \%$ due to obstructive nephropathy.

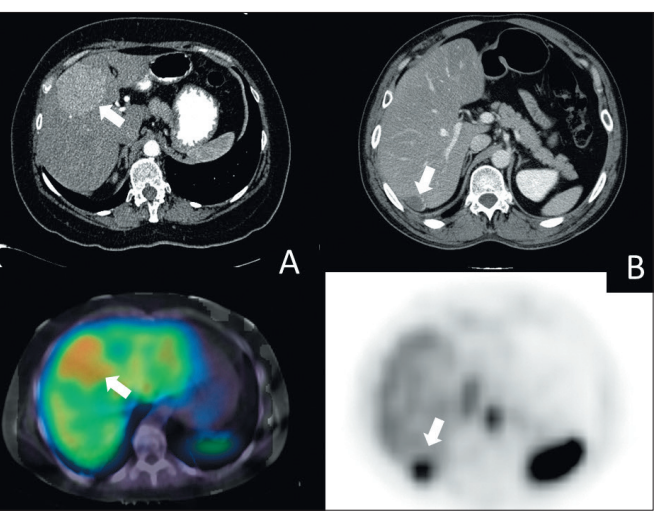

Fig. 7. Different cases showing liver lesions on CT with characterisation being confirmed as (A) focal nodular hyperplasia with increased [Tc-99m] colloid uptake, (B) and as haemangioma with intense [Tc-99m]-labelled red blood cell uptake.

\section{$B$}

\footnotetext{
Fig 